\title{
Density Functional Theory modeling of the structural and isotopic properties of $\mathrm{ZnO}$ surfaces
}

\author{
JIHAN LUBANI ${ }^{1}$, MARC BLANCHARD ${ }^{2}$, MERLIN \\ MÉHEUT $^{3}$, SIMONA FANTACCHI ${ }^{4}$ AND FILIPPO DE \\ ANGELIS $^{5}$
}

${ }^{1}$ Laboratoire Géosciences Environnement Toulouse (GET), CNRS, Université Toulouse III, IRD, CNES, 31400 Toulouse, France. Computational Laboratory for Hybrid/Organic Photovoltaics (CLHYO), CNR- SCITE), University of Perugia, 06123, Perugia, Italy.

${ }^{2}$ Géosciences Environnement Toulouse - CNRS - Univ Toulouse III - IRD - CNES - OMP

${ }^{3}$ CNRS Géosciences Environment Toulouse, Université de Toulouse, UPS, IRD, CNES

${ }^{4}$ CompuNet, Istituto Italiano di Tecnologia, Via Morego 30, 16163 Genova, Italy

${ }^{5}$ Computational Laboratory for Hybrid/Organic Photovoltaics (CLHYO), CNR- SCITE), University of Perugia, Via Elce di Sotto 8, 06123, Perugia, Italy. CompuNet, Istituto Italiano di Tecnologia, Via Morego 30, 16163 Genova, Italy.

Presenting Author: jihanloubani1@gmail.com

$\mathrm{ZnO}$ are very common systems present in various contexts and also model systems widely studied. $\mathrm{ZnO}$ nanoparticles are found in soil and water, it is therefore important to study the fate and behavior of this material in the environment, and isotopes are efficient tools for achieving this goal. Computer simulations can complement the direct materials analysis of these systems. In particular, we focused our theoretical study on the assessment of the surface effect on the isotopic signature. Do $\mathrm{ZnO}$ nanoparticles have different $\mathrm{Zn}$ isotope composition from bulk $\mathrm{ZnO}$ crystals in equilibrium conditions? In order to address this question, we performed structure and frequency calculations based on density functional theory (DFT) on various structural models. Isotopic properties are then determined from the vibrational frequencies. Three kinds of structural models were considered: $\mathrm{ZnO}$ bulk structure, the slab surfaces (10-10) and (21-10), and a model nanoparticle. The results obtained allow us to discuss the surface effect and the isotopic properties of $\mathrm{ZnO}$ nanoparticles. 